\title{
Invasive aspergillosis in a user of inhaled cocaine: rhinosinusitis with bone and cartilage destruction
}

\author{
Luna Azulay-Abulafia ${ }^{[1],[2],}$ Maria Auxiliadora Jeunon Sousa ${ }^{[2]}$, Andrea Pussanti ${ }^{[3]}$, \\ Daniel Dal'Asta Coimbra ${ }^{[4]}$, Hernando Vega ${ }^{[5]}$ and Fred Bernardes Filho ${ }^{[1]}$
}

[1]. Instituto de Dermatologia Professor Rubem Davi Azulay, Santa Casa de Misericórdia do Rio de Janeiro, Rio de Janeiro, RJ. [2]. Dermatologia, Universidade do Estado do Rio de Janeiro, Rio de Janeiro, RJ. [3]. Microbiologia, Clínica Privada, Rio de Janeiro, RJ. [4]. Dermatologia, Clínica Privada, Rio de Janeiro, RJ. [5]. Corporación Universitaria Rafael Nunez de Cartagena, Cartagena, Colombia.

\begin{abstract}
Aspergillosis is an infection caused by saprophytic fungi of the genus Aspergillus, which typically occurs in immunosuppressed individuals, but has also been reported in immunocompetent patients. The main routes of entry are the respiratory tract, skin, cornea, and ear, and the infection may be localized or disseminated by contiguity or vascular invasion. We report a severe case of rhinosinusitis with cutaneous involvement, caused by invasive aspergillosis, in an immunocompetent user of inhaled cocaine. Invasive aspergillosis related to cocaine abuse has not yet been reported in the literature. After itraconazole treatment and surgical debridement, complete clinical remission was achieved. Nasal reconstruction with a skin graft over a silicone prosthesis resulted in a satisfactory esthetic outcome.
\end{abstract}

Keywords: Aspergillosis. Aspergillus flavus. Rhinosinusitis. Cocaine addiction.

\section{INTRODUCTION}

Aspergillosis is an infection caused by saprophytic fungi of the genus Aspergillus, which typically occurs in immunosuppressed patients ${ }^{1,2}$. The main routes of entry are the respiratory tract, skin, cornea, and ear, and the infection may be localized or disseminated by contiguity or vascular invasion ${ }^{2,3}$. Patients with an impaired immune system or with disrupted anatomical and physiological barriers are at a high risk of developing the invasive form of the disease, characterized by fungal hyphae in tissues, which is associated with high mortality rates ${ }^{4}$.

Rhinosinusitis caused by Aspergillus is clinically similar to bacterial sinusitis. It rarely affects immunocompetent patients ${ }^{1,5}$. It was described in young individuals with predisposing factors to fungal colonization and proliferation, such as atopia; nasal polyps; and a history of allergic rhinitis, asthma, and recurrent sinusitis $^{2}$. In more severe cases, tissue necrosis and destruction may occur ${ }^{2,5}$. In a recent study regarding the distribution of different Aspergillus species in chronic sinus invasion, it was observed that Aspergillus flavus was the most predominant species worldwide, with the exception of the United States, where Aspergillus fumigatus was found to predominate ${ }^{6}$.

Address to: Dr ${ }^{\mathrm{a}}$ Luna Azulay-Abulafia. Av. Alexandre Ferreira 206, Lagoa, 22470-220 Rio de Janeiro, RJ, Brasil.

Phone: 5521 2537-2611

e-mail: lunaazulay@gmail.com

Received 15 April 2014

Accepted 9 May 2014
Cocaine abuse may lead to chronic rhinosinusitis; it can result in soft tissue and bone destruction, due to ischemia secondary to vasoconstriction, chemical irritation, trauma, changes in mucociliary mechanism, secondary infections, and local decrease in humoral and cellular immunity ${ }^{7,8}$. Cocaine users are predisposed to bacterial and fungal infections, such as aspergillosis. We report a case of rhinosinusitis with severe nasal damage and cutaneous involvement, secondary to cocaine inhalation, in an immunocompetent patient diagnosed with locally invasive aspergillosis (IA).

\section{CASE REPORT}

A 28-year-old female patient from Rio de Janeiro, who was a user of inhaled cocaine for the last six months, reported an erythematous lesion in the nose, which evolved with septal ulceration and perforation. The patient could not estimate the time of lesion emergence; she had no fever or other symptoms at presentation and had no history of rhinosinusitis.

A clinical examination revealed perinasal erythema and extensive damage of the columella, septum, and nasal floor, which was covered with a greenish-yellow material (Figures 1A and B). A biopsy sample obtained from the nasal floor showed extensive necrosis with several hyphae when analyzed with hematoxylin-eosin stain and Grocott's methenamine silver stain (Figure 2A). Culture analysis using Sabouraud's agar allowed the growth of an A. flavus colony (greenish-yellow, with a velvet texture, and transparent reverse). Microscopic analysis of the fungal colony showed long and grouped conidiophores with thick and clear walls. Globous and fertile (phialides) vesicles were observed in virtually all the fields (Figure 2B). 

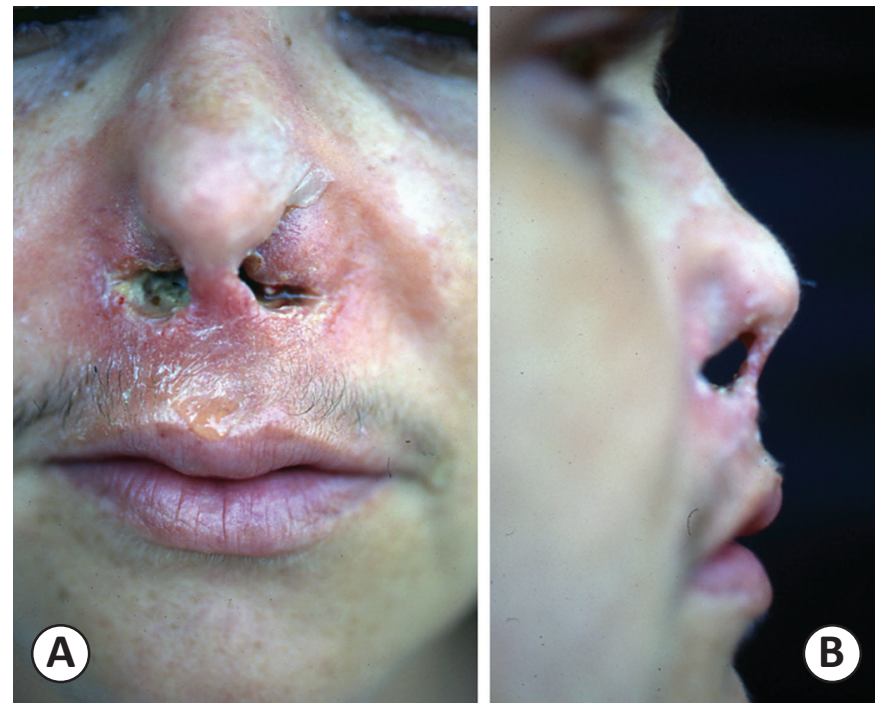

FIGURE 1 - A: Erythema and infiltration of the nose. B: A greenyellowish material on the nasal floor along with septal ulceration and perforation.

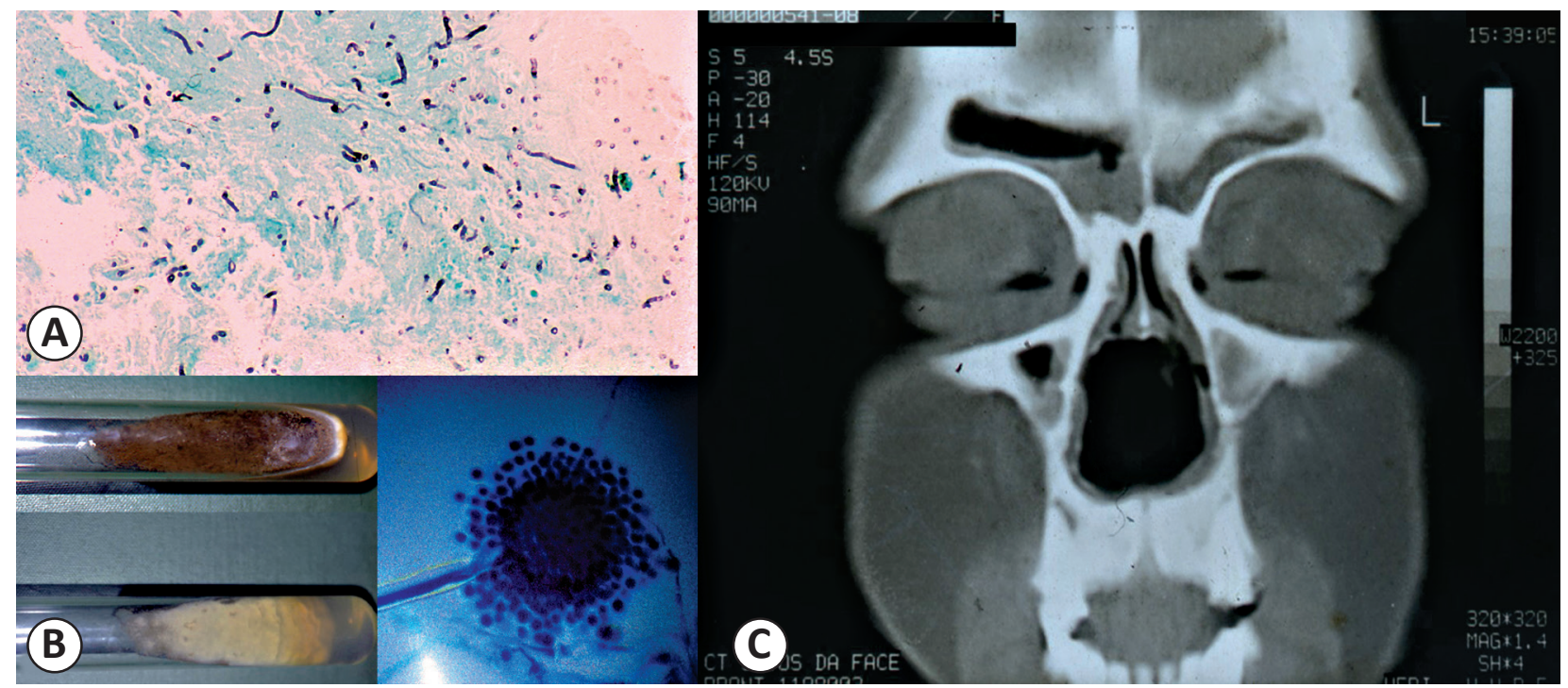

FIGURE 2 - A: Grocott stain analysis of the tissue sample obtained from the nasal floor. Numerous, septated, regular, branching hyphae are observed within the necrotic tissue, devoid of inflammation (100× magnification). B: Left. A greenishyellow colony, velvet texture, transparent reverse. Right. Microscopic analysis shows long and grouped conidiophores with thick and clear walls. Globous and fertile (phialides) vesicles are observed. C: Computed tomography of the face: extensive damage of the nasal septum and turbinates, except for the superior turbinate, and thinning and erosion of the septum and the internal maxillary sinus wall, communicating with the left nasal antrum and nasal fossa.

Complementary tests, including T cluster of differentiation 4 (CD4) ${ }^{+}$ and $\mathrm{CD}^{+}$cell counts, showed normal results. Result of the serology test was negative for anti-human immunodeficiency virus (HIV).

Computed tomography (CT) of the face revealed extensive damage of the nasal septum and turbinates, except for the superior turbinate, and thinning and erosion of the septum and the internal maxillary sinus wall, communicating with the left nasal antrum and nasal fossa. Opacity and edema of the mucosa were observed in the frontal, ethmoidal, and maxillary sinuses (Figure 2C).

Treatment consisted of itraconazole $400 \mathrm{mg} /$ day for 10 months and surgical debridement. Clinical and radiological improvement was observed in the patient, who later underwent surgical repair of the nasal cavity and achieved good esthetic results (Figure 3). 


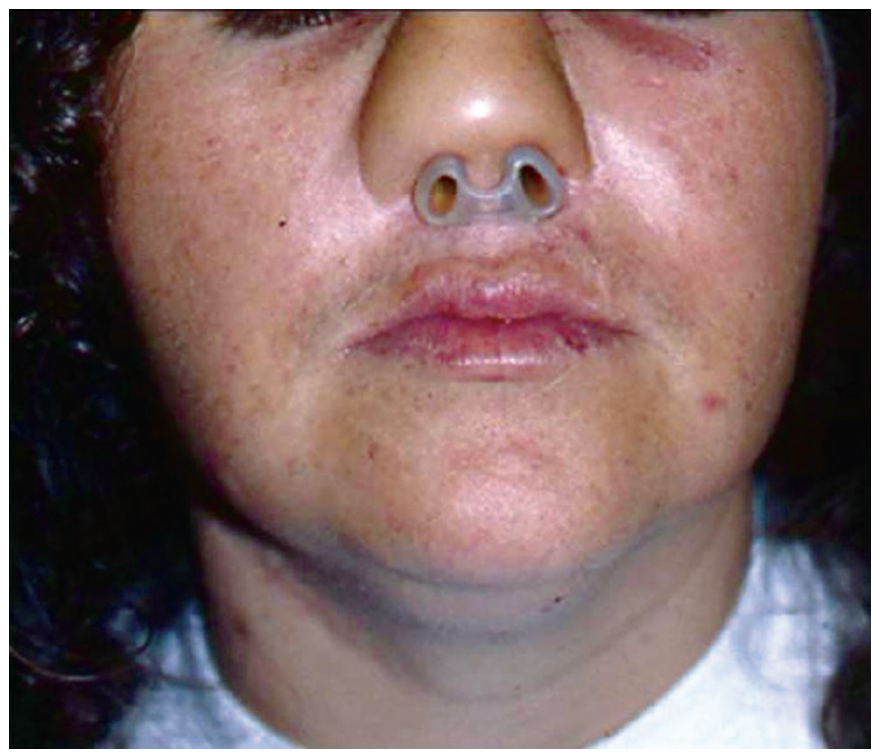

FIGURE 3 - Nasal reconstruction with a skin graft over a silicone prosthesis showing a satisfactory esthetic outcome.

\section{DISCUSSION}

Although chronic invasive fungal rhinosinusitis is uncommon in immunocompetent patients, such cases have been reported, particularly in Sudan and India, where it appears to be relatively common ${ }^{9}$. In Brazil, the few reported cases involved immunosuppressed patients ${ }^{10}$. To our knowledge, this is the first case of chronic invasive fungal rhinosinusitis in an immunocompetent Brazilian patient. This patient was a user of inhaled cocaine, and it is possible that cocaine abuse caused bone and cartilage destruction, facilitating fungal penetration ${ }^{2}$.

Few studies have reported the effect of IA on the central portion of the face in immunocompetent individuals ${ }^{1,2,4,5,11}$. Thus far, no study has reported its occurrence in relation to cocaine abuse.

Cocaine abuse in combination with the fungal infection led to severe rhinosinusitis and extensive destruction of the bone, internal maxillary sinus wall, cartilaginous and mucosal tissues, as well as invasion of the skin. This destructive effect was facilitated by continued cocaine abuse even after the initial clinical manifestation.

The usual presentation of fungal rhinosinusitis is nasal obstruction, disturbed smell, and postnasal drip associated with a nasal mass or polyp; it may be associated with orbital and intracranial invasion ${ }^{10}$.

In this case, IA was diagnosed by histopathologic examination, mycological culture analysis, and CT imaging. Cocaine triggered tissue destruction and probably local immunosuppression, contributing to pathogenic proliferation of opportunistic fungi, such as Aspergillus.

There has been increasing interest in preemptive antifungal therapy, which is defined as treatment that is deferred until an invasive fungal infection (IFI) can be diagnosed on the basis of substantive evidence. Early detection of diagnostic markers of a fungal infection, such as fungal nucleic acids, antigens, antibodies, or cell wall components, is essential in this regard. Galactomannan is an important diagnostic marker used in the diagnosis of IA. Another serum marker commonly used to indicate the presence of IFIs is (1-3)-b-D-glucan (BDG). However, galactomannan or beta-glucan assays are not easily available, and conventional direct microscopy, histopathology, and culture analysis are the only available techniques in most centers for the diagnosis of $\mathrm{IA}^{10}$.

Both CT and magnetic resonance (MR) images help to determine the clinical diagnosis, recurrence rate, and delineation of the extension of fungal sinusitis as well as in surgery ${ }^{10}$. Fungal sinusitis should be considered in all patients with chronic sinusitis, especially when it occurs in association with certain clinical features like intractable symptoms, despite adequate treatment for bacterial sinusitis, allergic rhinitis, asthma, nasal polyposis (non-invasive types) or fever, headache, epistaxis, diabetes, nasal mucosal ulcer, orbital apex syndrome, or proptosis (invasive types). However, the diagnosis of fungal sinusitis depends on direct microscopic examination, culture, and histopathological analysis of tissue or the cheesy material obtained from sinuses ${ }^{9,10}$.

In our patient, etiological investigation was performed by fungal and bacterial culture analyses of the greenish-yellow material found on the nasal floor, which was removed by surgical debridement. Hyphae were observed in the tissue sample, and the mycological culture revealed the presence of A. flavus. The CT image showed extensive damage of the nasal septum and turbinates, with some exception.

Oral itraconazole was chosen to manage the patient because the patient was immunocompetent; moreover, it was the only option available for the patient at the hospital where she was treated. Since the initial response was satisfactory, treatment remained unchanged until complete clinical remission. The excellent response to itraconazole further confirmed the presence of a fungal infection. In the literature, even for immunocompetent patients, other therapeutic approaches have been proposed, such as intravenous amphotericin B followed by itraconazole and voriconazole followed by itraconazole ${ }^{12}$.

It is possible that the inhalational form of cocaine used by the patient was contaminated with the fungus. We suggest that inhaled cocaine abuse could be one of the predisposing factors of IA in immunocompetent patients.

\section{FINANCIAL SUPPORT}

Research performed in Hospital Universitário Pedro Ernesto da Universidade do Estado do Rio de Janeiro (HUPE/UERJ), Rio de Janeiro, Brazil.

\section{REFERENCES}

1. Khatri ML, Stefanato CM, Benghazeil M, Shafi M, Kubba A, Bhawan J. Cutaneous and paranasal aspergillosis in an immunocompetent patient. Int J Dermatol 2000; 39:853-856. 
2. Agarwal S, Kanga A, Sharma V, Sharma DR, Sharma ML. Invasive aspergillosis involving multiple paranasal sinuses, a case report. Indian J Med Microbiol 2005; 23:195-197.

3. Woodruff CA, Hebert AA. Neonatal primary cutaneous aspergillosis: case report and review of the literature. Pediatr Dermatol 2002; 19:439-444.

4. Knipping S, Holzhausen HJ, Koesling S, Bloching M. Invasive aspergillosis of the paranasal sinuses and the skull base. Eur Arch Otorhinolaryngol 2007; 264:1163-1169.

5. Subramanian S, Kandpal H, Sharma R, Pushkar N, Sen S, Gamanagatti S, et al. Invasive sinus aspergillosis with perineural spread in an immunocompetent patient. Australas Radiol 2007; 51 (supl):B189-192.

6. Webb BJ, Vikram HR. Chronic invasive sinus aspergillosis in immunocompetent hosts: a geographic comparison. Mycopathologia 2010; 170:403-410

7. Trimarchi M, Nicolai P, Lombardi D, Facchetti F, Morassi ML, Maroldi R, et al. Sinonasal osteocartilaginous necrosis in cocaine abusers: experience in 25 patients. Am J Rhinol 2003; 17:33-43.
8. Simsek S, de Vries XH, Jol JA, Spoelstra-de Man AM, Nanayakkara PW, Smulders YM, et al. Sino-nasal bony and cartilaginous destruction associated with cocaine abuse, S. aureus and antineutrophil cytoplasmic antibodies. Neth J Med 2006; 64:248-251.

9. Yagi HI, Gumaa SA, Shumo AI, Abdalla N, Gadir AA. Nasosinus aspergillosis in Sudanese patients: clinical features, pathology, diagnosis, and treatment. J Otolaryngol 1999; 28:90-94.

10. Chakrabarti A, Chatterjee SS, Das A, Shivaprakash MR. Invasive aspergillosis in developing countries. Med Mycol 2011; 49 (suppl 1):S35-47.

11. Kaya S, Yavuz I, Cobanoğlu U, Ural A, Yılmaz G, Köksal I. Fatal sinoorbital aspergillosis in an immunocompetent case. Mikrobiyol Bul 2011; 45:546-552.

12. Pushker N, Meel R, Kashyap S, Bajaj MS, Sen S. Invasive aspergillosis of orbit in immunocompetent patients: treatment and outcome. Ophthalmology 2011; 118:1886-1891. 